\title{
Comparison of cinefluoroscopy and $M$ mode echocardiography for detecting aortic valve calcification Correlation with severity of stenosis of non-rheumatic aetiology
}

\author{
MARK DANCY, GRAHAM LEECH, AUBREY LEATHAM \\ From the Cardiac Department, St George's Hospital, London
}

SUMMARY The density of aortic valve calcification was estimated using cinefluoroscopy and M mode echocardiography in 86 patients with pure aortic stenosis. The results were compared with the degree of outflow obstruction measured haemodynamically. Cinefluoroscopic estimates of aortic valve calcification correlated well with measured aortic valve gradient whereas echocardiographic results were less accurate. Echocardiography gave accurate information about the distribution of calcium within the cusps, but this was not of value in predicting the degree of obstruction.

The amount of calcium in the aortic valve as assessed by simple cinefluoroscopy is a useful guide to the severity of aortic stenosis in patients in the middle and older age groups.

Calcification of the aortic valve is an almost invariable feature of aortic stenosis in patients over the age of 50 years. ${ }^{1}$ This fact has been recognised ever since the earliest descriptions of aortic stenosis, ${ }^{2}$ and the search for calcium has become an accepted part of the clinical assessment of aortic stenosis. The simplest method of viewing calcium in the aortic valve is by lateral chest radiography. ${ }^{3}$ Cinefluoroscopy is also of value in assessing aortic stenosis, ${ }^{34}$ but little is known of the value of echocardiographic estimates of calcification.

The object of this study was to estimate the degree of calcification of the aortic valve in patients with pure aortic stenosis using both $M$ mode echocardiography and cinefluoroscopy and to compare these estimates with the degree of outflow obstruction. The distribution of calcium was also assessed and compared with radiographs of excised aortic valves.

\section{Patients and methods}

Eighty six patients over the age of 30 years were studied. All were considered to have aortic stenosis on clinical grounds. Aortic regurgitation, if present, was haemodynamically insignificant. No patient had clini-

Requests for reprints to Dr Mark Dancy, Cardiac Department, St George's Hospital, Blackshaw Road, London SW17 0QT. cal or echocardiographic evidence of mitral valve disease, and all those who underwent surgery had normal mitral valves. Cineangiograms were available for $\mathbf{8 0}$ patients. All of these were analysed, although some were of poor quality. Four echocardiograms were considered to be uninterpretable, and this left 82 recordings for assessment. Calcification of the aortic valve was graded by reference to the criteria in the Table. Fig. 1 shows an example of an echocardiogram in each category.

In addition to grading the degree of calcification, any echocardiogram in which there appeared to be an unusual distribution of calcium was carefully analysed, particularly when the calcium appeared to be confined to one cusp since this may possibly indicate mild stenosis.

Results were compared with the peak systolic gradient across the aortic valve measured at cardiac catheterisation.

Results

PATHOLOGY OF THE AORTIC VALVES

The pathology of the aortic valve was known in 55 cases. Forty nine patients had bicuspid valves and five had tricuspid valves. Although the inclusion criteria were expected to exclude patients with rheumatic disease, one patient was thought to have a rheumatic 


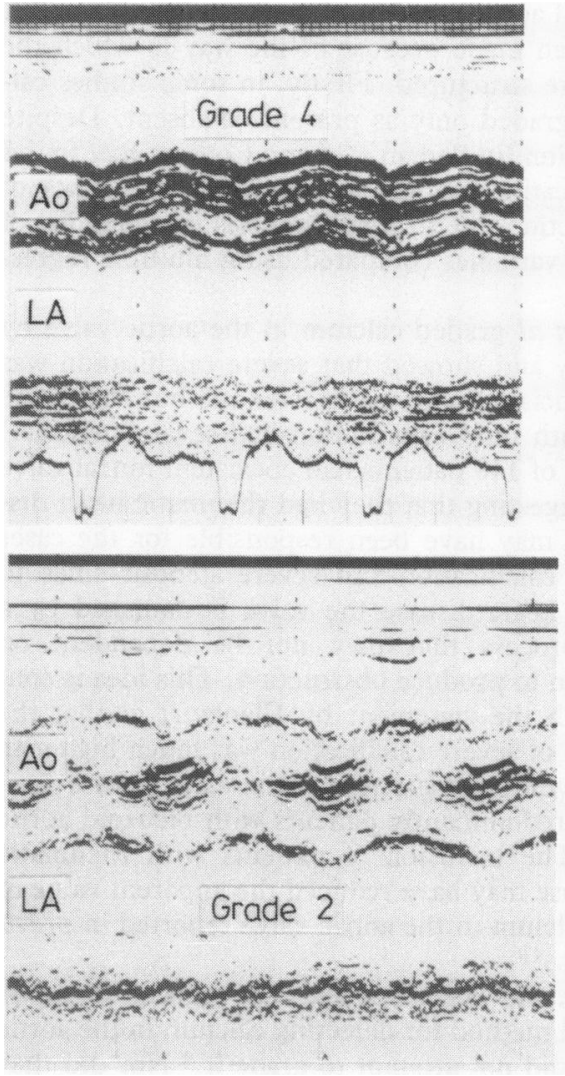

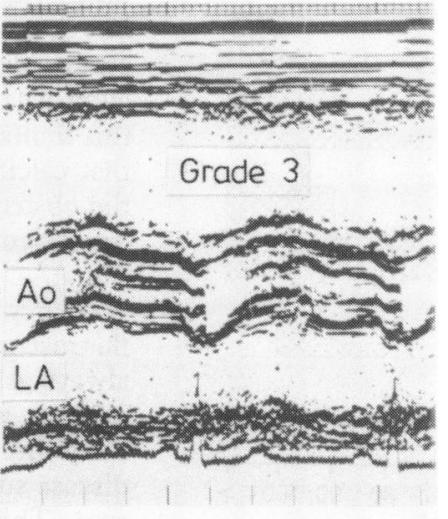

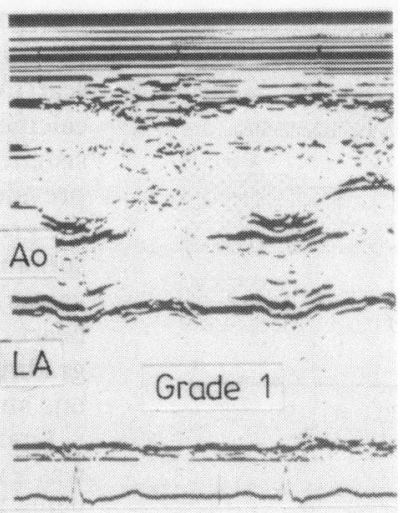

Fig. 1 Examples of echocardiograms showing different grades of calcification of the aortic valve. Ao, aorta; $L A$ lefi atrium. (See Table for the criteria for grading.) valve at operation. The pathology was uncertain in the remaining patients, but most of them probably had bicuspid valves since none had evidence of mitral valve disease either on echocardiography or at catheterisation.

\section{GRADING OF CALCIUM}

The number of patients with each grade of aortic valve calcification as determined by cinefluoroscopy and echocardiography is shown in Fig. 2. This is plotted against the gradient on the horizontal axis to show the relation between the degree of calcification and the outflow obstruction.
There was a good association between the fluoroscopic grading of calcium and the gradient measured at cardiac catheterisation. Nevertheless, the grading of calcium by echocardiography showed little association with the gradient, and one grade (grade 2) appeared much more frequently than would be expected by chance.

\section{DISTRIBUTION OF CALCIUM}

The distribution of calcium was difficult to determine using fluoroscopy since the aortic walls were not defined. Only when a catheter was placed between the aortic leaflets was there enough spatial information

Table Criteria for grading calcification by cineftuoroscopy and echocardiography

\begin{tabular}{lll}
\hline Grade & Criteria & \\
\cline { 2 - 3 } & Fluoroscopic & Echocardiographic \\
\hline 0 & $\begin{array}{l}\text { No calcium } \\
\text { Minor flecks of calcium }\end{array}$ & $\begin{array}{l}\text { Normal density of echoes } \\
\text { Increased density of echoes from valve }\end{array}$ \\
2 & $\begin{array}{l}\text { Definite calcium present but not throughout valve } \\
\text { Whole valve outlined by calcium }\end{array}$ & $\begin{array}{l}\text { Fairly heavy echoes from valve and aortic wall } \\
\text { Dense echoes beginning to fill the aorta, often clearing } \\
\text { in systole }\end{array}$ \\
4 & Dense calcification & Whole aorta filled with dense echoes \\
\hline
\end{tabular}



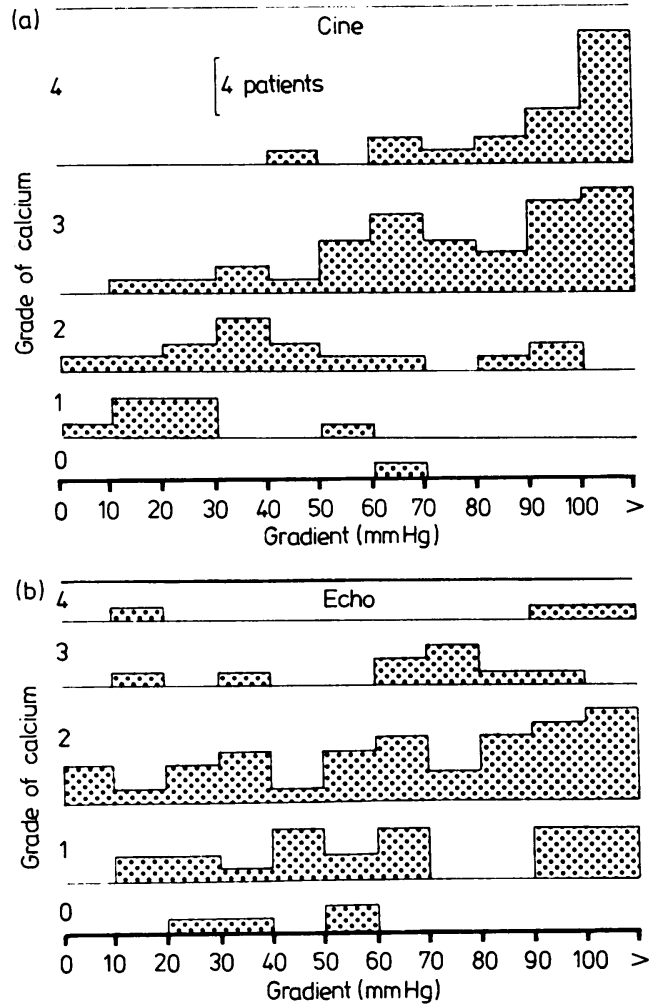

Fig. 2 Frequency histograms showing the number of patients with different grades of calcium in the aortic valve related to the gradient across the valve as assessed by (a) cinefluoroscopy and (b) echocardiography.

confidently to determine the site of the calcium.

With $M$ mode echocardiography there was better spatial information as the walls of the aorta could be defined. Unilateral calcification was found in $16 \%$ (13) of the patients. In nearly all of these (12) the calcium was anteriorly placed (Fig. 3), and in only one case was it posterior. The finding of unilateral calcification was of no help in predicting the gradient. Half of those patients with unilateral anterior calcification had gradients over $50 \mathrm{~mm} \mathrm{Hg}$ (range $20-140 \mathrm{~mm} \mathrm{Hg}$ ). The patient with unilateral posterior calcification had a gradient of $60 \mathrm{~mm} \mathrm{Hg}$.

\section{Discussion}

ASSESSMENT OF THE DEGREE OF CALCIFICATION A number of studies have drawn attention to the importance of calcification of the aortic valve in the diagnosis of aortic stenosis, ${ }^{3-7}$ but full use of this fact has not been made because of the way in which the studies were structured. Firstly, in some studies calcium was graded only as present or absent. Despite this limitation Eddleman et al and Cousins et al found that calcification was the most powerful predictor of the obstruction due to aortic stenosis in a long list of dependent variables compared using multiple regression. ${ }^{67}$

Glancy et al graded calcium in the aortic valve by fluoroscopy and showed that severe calcification was always associated with severe stenosis but that some patients with mild calcification had severe stenosis. ${ }^{3}$ Fifty three of 148 patients had coexistent mitral valve disease suggesting that they had rheumatic heart disease. This may have been responsible for the cases with mild calcification and severe stenosis since in rheumatic heart disease the valve is damaged by a scarring process that may not be dependent on calcification to produce obstruction. This idea is consistent with the statement by Glancy et al that the prevalence of severe calcification was much higher in the group without coexistent mitral valve disease (presumably predominantly patients with bicuspid aortic valves). ${ }^{3}$ The inclusion of patients with rheumatic heart disease may have reduced the apparent value of grading calcium in the aortic valve reported in previous studies. 58

Gramiak and Shah reported that echocardiography was a good method for detecting calcium in the aortic valve but did not attempt to grade it. ${ }^{9}$ Nor did they study the pathology of the valves.

The results of the present study show that grading the extent of calcium deposition by cinefluoroscopy is extremely valuable. Only one of the 20 patients with severe calcification by this method had a gradient of less than $50 \mathrm{~mm} \mathrm{Hg}(45 \mathrm{~mm} \mathrm{Hg})$. One patient with no calcification on fluoroscopy had severe stenosis, but his cineangiogram was of poor quality and the surgically excised valve was in fact extensively calcified.

The intermediate grades 2 and 3 were the most difficult to separate visually, and this is reflected in some overlap in Fig. 2, but it was still considered valuable to attempt to separate these grades as it was at this level that the clinically important watershed between mild and severe aortic stenosis was found.

The system of grading of calcium by echocardiography was much more difficult, and we were less confident when applying it. There was no clear relation between the degree of calcium on echocardiography and the gradient, and there was a distinct preference for grade 2 suggesting either that the observer was biased or more likely that the recorder of the echocardiogram tended to adjust the brightness of the echoes by means of the gain control in order to produce an "acceptable" picture. 


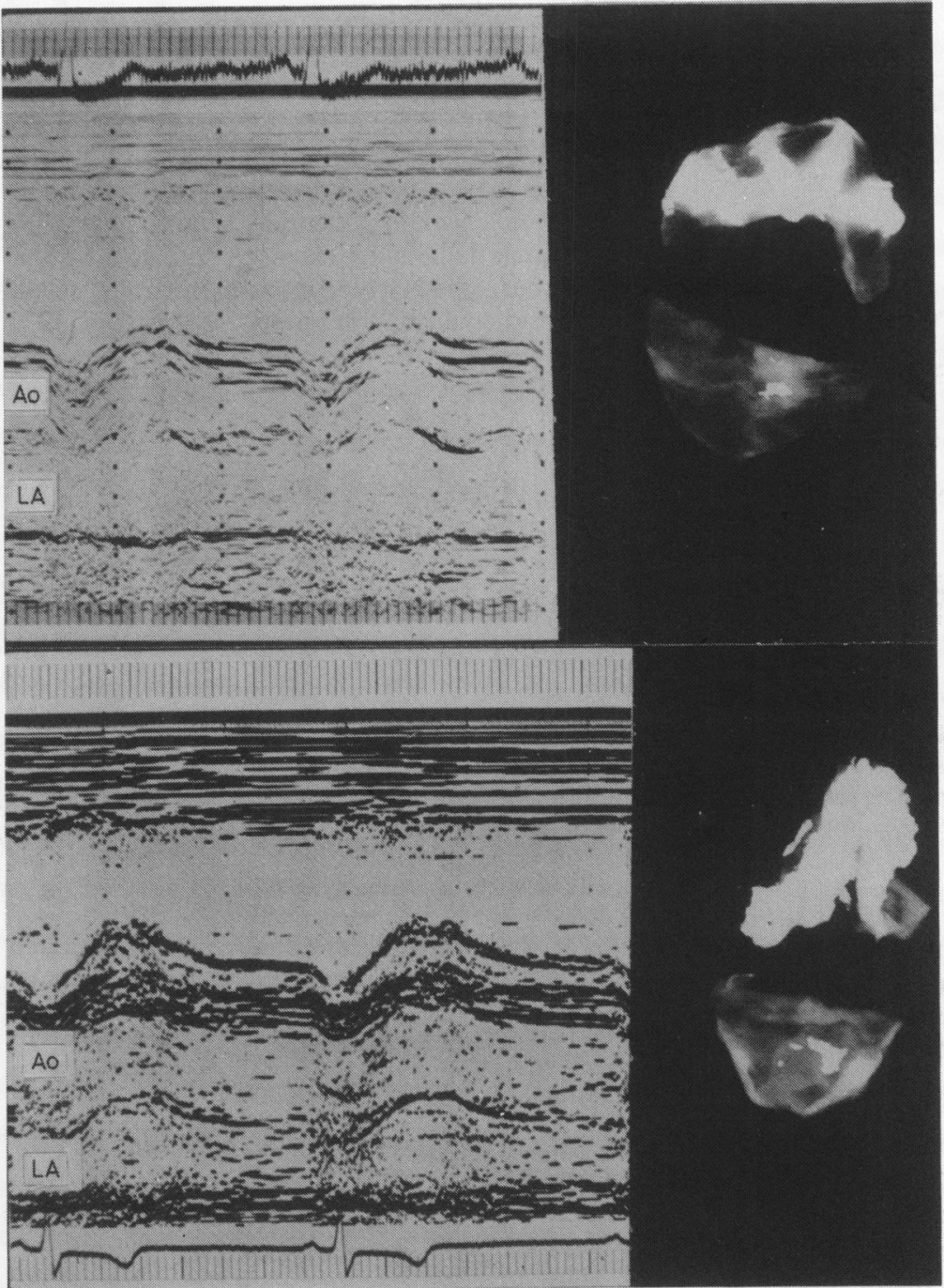

Fig. 3 Examples of echocardiograms from two patients with the radiograph of the excised valve on the right. Both show unilateral calcification of the anterior cusp, but both valves produced severe obstruction. Ao, aorta; LA, left atrium.

\section{DISTRIBUTION OF CALCIUM}

Although the echocardiogram gave better spatial orientation than cinefluoroscopy, and was able to identify unilateral calcification, this was not useful information for predicting the severity of the stenosis. One of the possible explanations for this is that the echocardiogram was giving false information about the actual distribution of calcium. Nevertheless, when the excised valves were examined radiographically there was good agreement between the echocardiogram and the radiographic appearances (Fig. 3). This suggests that calcification of just one leaflet of a bicuspid valve may be sufficient to produce severe stenosis. All the patients in this study with severe aortic stenosis had at least some calcium in their aortic valve.
Nevertheless, a case of tricuspid aortic stenosis with no calcium even on a direct radiograph of the aortic valve has recently been reported. The echocardiogram of this patient is shown in Fig. 4. When the valve was inspected visually it was stiff and thickened by amorphous material with no particular staining characteristics. This case was not included in the present series.

The underlying pathology is clearly inportant in determining whether the degree of calcification of the aortic valve is closely related to the stenosis. As already mentioned, there is evidence that rheumatic aortic valves can become stenosed without calcifying, and the same appears to be true occasionally in cases of tricuspid aortic stenosis. Nevertheless, the present 


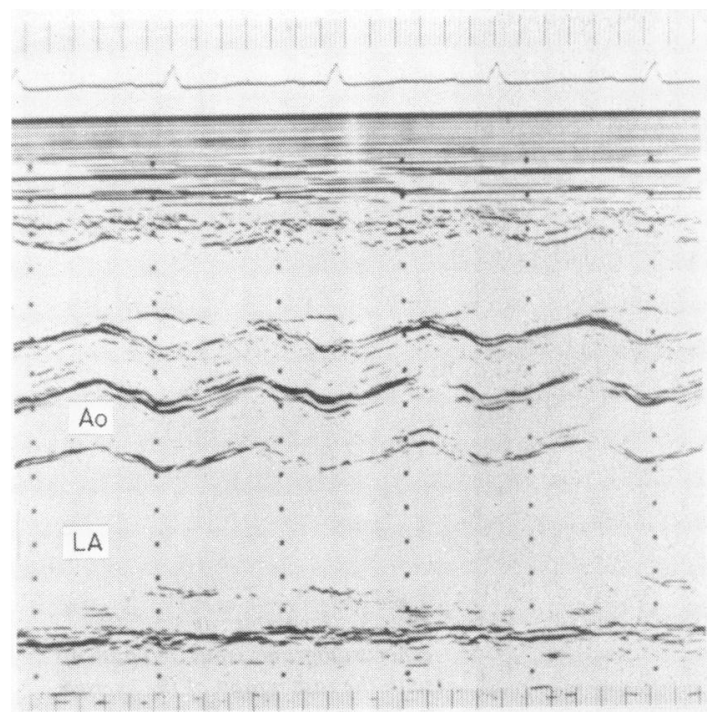

Fig. 4 Echocardiogram of a 70 year old patient with severe aortic stenosis and little or no calcium in the aortic valve on the echocardiogram. She was found to have severe aortic stenosis. The excised valve was tricuspid and contained no calcium when examined radiographically. Ao, aorta; $L A$, left atrium.

series suggests that in patients over the age of 30 years with bicuspid valves the development of stenosis is dependent on deposition of calcium in the cusps and, therefore, that the degree of calcification as judged by cinefluoroscopy is closely related to the orifice area of the valve.

The echocardiogram appears, therefore, to give information on the distribution of calcium but not to be as accurate as cinefluoroscopy in differentiating degrees of calcification. The differential absorption of $x$ rays by calcium is such that there is rarely any doubt when calcium is present using an image intensifier. On the other hand, with echocardiography similar appearances can be seen from both fibrosed leaflets and calcium, the only difference being the intensity of the echoes. As there is no standardisation of gain controls the echoes from a thickened valve may be sufficiently intensified for it to appear calcified and vice versa. Perhaps with the new generation of ultrasound machines incorporating facilities for tissue characterisation ${ }^{10}$ this problem will be resolved, but until then it will remain difficult using either $M$ mode or cross sectional echocardiography. ${ }^{11}$

Cinefluoroscopy is a simple technique which is easily carried out in an outpatient department. Although most hospitals do not have extensive cardiac investigation facilities, good screening equipment is widely available and could be used more for this purpose.

\section{References}

1 Campbell M. Calcific aortic stenosis and congenital bicuspid aortic valves. Br Heart $\mathcal{f}$ 1968; 30: 606-16.

2 Cowper W. Of ossifications or petrifications in the coats of arteries, particularly in the valves of the great arteries. Philosophical Transactions of the Royal Society London 1706; 24: 1970-7.

3 Glancy DL, Freed TA, O'Brien KP, Epstein SE. Calcium in the aortic valve: roentgenologic and hemodynamic correlations in 148 patients. Ann Intern Med 1969; 71: 245-50.

4 Batson GA, Urquhart W, Sideris DA. Radiological features in aortic stenosis. Clin Radiol 1972; 23: 140-4.

5 Klatte EC, Tampas JP, Campbell JA, Lurie PR. The roentgenographic manifestations of aortic stenosis and aortic valvular insufficiency. AfR 1962; 88: 57-69.

6 Eddleman EE Jr, Frommeyer WB Jr, Lyle DP, Bancroft WH Jr, Turner ME Jr. Critical analysis of clinical factors in estimating severity of aortic valve disease. $\mathrm{Am} \mathcal{F} \mathrm{Car}$ diol 1973; 31: 687-95.

7 Cousins AL, Eddleman EE Jr, Reeves TJ. Prediction of aortic valvular area and gradient by noninvasive techniques. Am Heart $\mathcal{f}$ 1978; 95: 308-15.

8 Mitchell AM, Sackett CH, Hunzicker WJ, Levine SA. The clinical features of aortic stenosis. Am Heart $\mathcal{f}$ 1954; 48: 684-720.

9 Gramiak R, Shah PM. Echocardiography of the normal and diseased aortic valve. Radiology 1970; 96: 1-8.

10 Gramiak R, Waag RC, Schenk EA, Lee PPK, Thomson $\mathbf{K}$, Macintosh P. Ultrasonic detection of myocardial infarction by amplitude analysis. Radiology 1979; 130: 713-20.

11 DeMaria AN, Bommer W, Joye J, Lee G, Bouteller J, Mason DT. Value and limitations of cross sectional echocardiography of the aortic valve in the diagnosis of valvular aortic stenosis. Circulation 1980; 62: 304-12. 